\title{
Not the first, not the best
}

\author{
Before accepting research manuscripts for publication, we ask authors to refrain from making priority or novelty \\ claims and to remove qualitative evaluations of their own work. Both policies are intended to increase the accuracy \\ and credibility of research we publish.
}

S ince the launch of the journal in 2017, it has been our policy that authors remove novelty/priority claims as a condition of formal acceptance for publication. In 2019, we introduced the requirement that authors avoid the use of self-laudatory language and qualitative characterizations of their work.

Priority, novelty, and extraordinary significance or outstanding research quality play a key role in science. However, the culture of 'being the first or the bestideally, both' provides strong incentives to authors to make such claims in research manuscripts, even when they are not warranted. Priority or novelty claims not only are subject to abuse, they cannot be formally verified for the vast majority of fields. Claims of exceptional quality or significance distract from the actual evidence, reduce the credibility of research, and are best left to readers, who are the ultimate judges of value and rigour.

Editors and reviewers at many journals, including Nature Human Behaviour, evaluate manuscripts on perceived conceptual or methodological novelty (among other potential contributions to scientific knowledge, such as an advance in evidence). However, the editorial and peer review process does not provide a guarantee or certification of novelty. Such judgments are subject to inevitable knowledge limitations imposed by a vast body of knowledge production that does not have a formal system of recording priority except via the act of publication itself. Removing priority

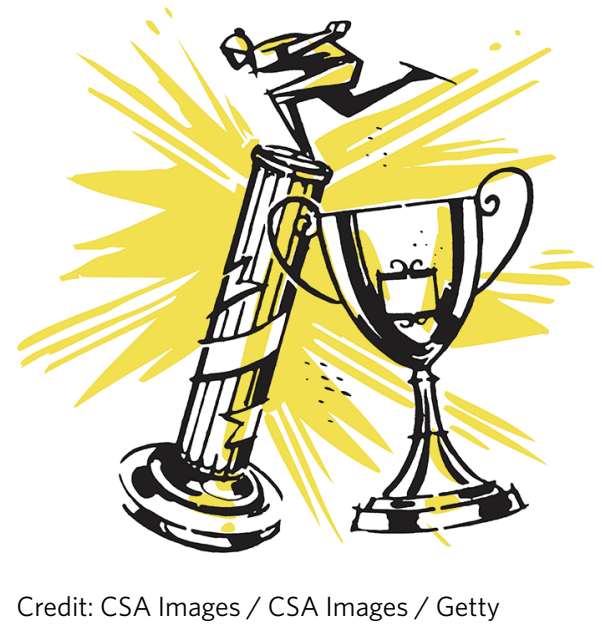

own work, and exaggerated claims of importance or rigour can backfire not only when editors and peer reviewers evaluate a manuscript, but more importantly, when a paper becomes a matter of public record and scrutiny.

Validation of any extraordinary attributes of a research project comes from readers and is enabled via detailed factual description of prior knowledge, current study design, evidence, and conclusions. Excessive use of adjectives and adverbs when describing the study's significance or rigour is frequently the main culprit. "An unprecedentedly massive and highly rigorous study that, crucially, provides a major step forward..." is best described instead as "a preregistered study with $n=x$ participants that provides a step toward...." If the study provides sufficient information on the prior literature and its own methods and sampling, as well as access to a time-stamped preregistered protocol, readers will not fail to appreciate any exceptional features of the work in comparison to its precedents. Nor will they potentially dismiss your work out of hand if the evidence fails to live up to extraordinary claims of significance but is still valuable nonetheless.

Letting the facts of your research project speak for themselves is the best policy unverifiable novelty or priority claims and self-laudatory language obscure what matters most: the evidence itself.

Published online: 18 February 2021 https://doi.org/10.1038/s41562-021-01068-X 